\title{
A Bi-Target Based Mobile Relay Selection Algorithm for MCNs
}

\author{
Huijun Dai ${ }^{1,2}$, Xiaolin Gui ${ }^{1,2}$, Zhaosheng Dai ${ }^{1,2}$, Dewang Ren ${ }^{1,2}$ and Yingjie Gu ${ }^{1,2}$ \\ 1 School of Electronic and Information Engineering, Xi'an Jiao tong University \\ Shaanxi,Xi'an,710049, PR.china \\ [e-mail: daihuijun@mail.xjtu.edu.cn] \\ 2 Shaanxi Province Key Laboratory of Computer Network, Xi'an Jiao tong University \\ Shaanxi,Xi'an,710049, PR.china \\ *Corresponding author: Xiaolin Gui
}

Received December 27, 2016; revised May 18, 2017; accepted June 24, 2017;

published November 30, 2017

\begin{abstract}
Multi-hop cellular networks (MCNs) reduce the transmit power and improve the system performance. Recently, several research studies have been conducted on MCNs. The mobile relay selection scheme is a rising issue in the design of MCNs that achieves these advantages. The conventional opportunistic relaying (OR) is performed on the single factor for maximum signal-to-interference-plus-noise ratio (SINR). In this paper, a comprehensive OR scheme based on Bi-Target is proposed to improve the system throughput and reduce the relay handover by constraining the amount of required bandwidth and SINR. Moreover, the proposed algorithm captures the variability and the mobility that makes it more suitable for dynamic real scenarios. Numerical and simulation results show the superiority of the proposed algorithm in both enhancing the overall performance and reducing the handover.
\end{abstract}

Keywords: Mobile relay, MCNs, relay selection algorithm, multi-objective optimization.

This research was supported in part by NSFC under grant no.61472316 andno.61172090,National Science and Technology Major Project under grant2012ZX03002001, Research Fund for the Doctoral Program of Higher Education of China under grant no.20120201110013,Scientific and Technological Project in Shaanxi Province under grant no.2012K06-30,no.2014JQ8322), Basic Science Research Fund in Xi'an Jiaotong University (no.XKJC 2014 008), Shaanxi Science and Technology Innovation Project(2013SZS16-Z01/P01/K01), And National Science Foundation of Jiangsu Province (BK20141214). 


\section{Introduction}

Multi-hop cellular networks (MCNs) have advantages in network coverage and information transmission rate and have become an attractive topic due to its low energy consumption in short distance [1]. The D2D (Device-to-Device) communication functionality of the MCNs allows the mobile nodes (MNs) to communicate with each other by using multi-hop forwarding. In order to improve the end-user Quality of Service (QoS) and the link quality of the MNs, the MNs with good cellular channel quality should directly communicate with the base station (BS). While the MNs with poor cellular channel quality, especially the cell-edge MNs, should indirectly communicate with the BS via relay nodes (RNs) in the vicinity [2] which are MNs with good cellular channel quality. The collaborative communication using the RNs is capable of efficiently combating wireless impairments and coverage extension. In a real case scenario, each node is surrounded by some other nodes with different channel state information (CSI) [3] and relative positions as well as different moving directions. The proper selection of a node as a relay will influence several aspects of the collaborative communication [4]. In MCNs, the MNs with good CSI are named as one-hop nodes or one-hop users [5] and the links between the MNs and the BS are labeled as direct links (DLs). The other nodes with poor channel quality at the cell-edge are named as the double-hop nodes or double-hop users. In two-hop scenarios, the double- hop nodes select some relays as the cooperation nodes. The link between the double-hop node and the relay node is labeled as the access link (AL) and the link between the relay and the BS is termed as the backhaul link (BL)[3] (see Fig. 1).

In fact, the mobile opportunistic relaying (OR) is used to balance the channel quality between the $\mathrm{AL}$ and the $\mathrm{BL}$ with maximizing the transmission while fulfilling the prioritized cellular service constraints [1]. Many related studies have been conducted on mobile opportunistic relays due to their advantages of reduced transmit power, improved system capacity and network coverage [6][7]. While others have been presented to promote the communication rate, enhance the achievable rate or the system throughput [8]. Some research analyzed spectrum prediction, eg.in [9] [10] [11], which is a challenging problem. The optimizing targets involve two categories: maximal terms such as the system throughput, the signal-to-interference-plus-noise ratio (SINR) [12], the wireless frequency spectrum utilization [9] [10], and minimal terms such as the energy consumption, the relative interference, the link error and the number of switching.

Actually, the selection strategy must consider the relay switching as an important issue especially when the MCNs or mobiles number is huge [13]. Frequent relay switching involves handover from one $\mathrm{RN}$ to others that complicates the implementation of the relay selection in two-hop scenarios [14]. Meanwhile, it causes two technical bottlenecks: the system 
throughput and the switching rate. It should be mentioned that in the works aforementioned, it was assumed that the moving node with low speed will not cause switching [15]. However, in practical city microcells environments, especially in shadow area, the moving nodes pattern predicting will influence the switching accordingly.

The remainder of this paper is organized as follows: Section II presents the mobile scenario that constitutes the general elements; A relay selection scheme based on bi-target selection is proposed and analyzed in Section III, bi-target means optimizing the system throughput and the relay switching fulfilling the fixed constraints; The functionality of the proposed strategy is described in Section IV; The performances of schemes are evaluated through a system-level simulation in Section V; Lastly, this study is concluded in Section VI.

\section{Model of Mobile Scenario in MCNs}

The MCNs is divided into cells with a BS at the center in a model of mobile scenario. The location of the mobile relay is changing over time and the mobile users or the MNs can communicate with the BS via AL, BL or DL [14] (see Fig. 1). However, the dense distribution of urban buildings will result in the appearance of signal blind area (see grey region in Fig. 1). Widespread MNs have several choices in relay section. In this paper, the $\mathrm{RN}$ in the cell-shadow regions is chosen as an example.

As shown in Fig. 1, the center node $O(0,0)$ is the location of the BS and the covering radius is $R$. The shadow is viewed as an approximate circle with $s\left(x_{s}, y_{s}\right)$ and $r_{s}$ as the center and the radius of the shadow, respectively. The node $M N_{1}\left(x_{m}, y_{m}\right)$ in the shadow region has communication range with radius $r_{0}$. It chooses a free node $R N_{1}\left(x_{r}, y_{r}\right)$ nearby as relay to communicate with the BS. According to the technical report [16] during the 3rd Generation Partnership Project (3GPP) standards development, the physical layer parameters will be decided using the system scenarios. The path loss (PL) in the urban macro-cell size of 36.942 is formulated in formula 1 with $d$ in meters [16]:

$$
\begin{gathered}
\operatorname{SINR}_{\text {Cell }}=\frac{P_{B S} \cdot G_{B S} \cdot G_{\text {loss }}{ }^{-1} P L(d)^{-1}}{N_{0}+I_{0}} \\
P L_{d B}=128.1+37.6 \lg \frac{d}{1000}
\end{gathered}
$$




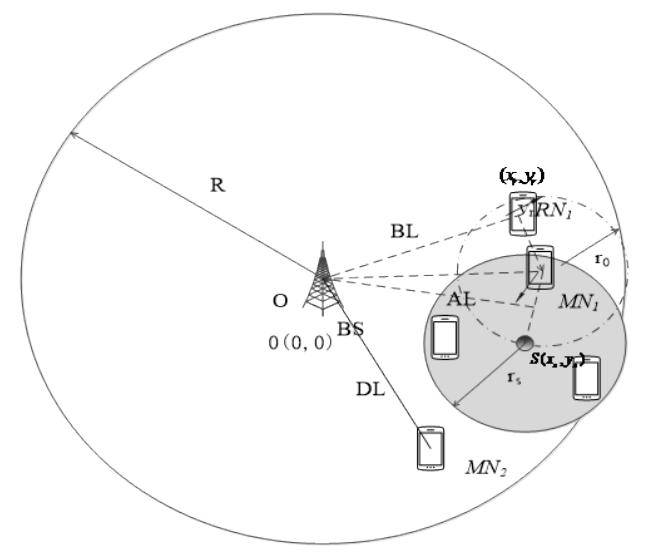

Fig. 1. MNs in the city microcell shadow region.

In formula 1 and 2, $N_{0}, I_{0}$ are the interference power and the noise power, respectively, and $P_{B S}, G_{B S}$ are the transmitting power and the antenna gain of the BS. $d$ is the distance from RN to BS or from MN to RN. PL is the path loss between RN and BS or between MN and RN. The SINR Cell is measured, calculated and compared with the threshold. Then the one-hop and the double-hop nodes are separated and consequently the radiuses of different links in Fig. 1 are calculated.

\subsection{Node Motion Patters}

The motion patters of MN also have two categories: random walk mobility (RWM) and boundless area mobility (BAM) [17]. For RWM, the moving velocity and the direction of the MNs are random and they stop or move randomly. In BAM, the MNs' current state has close correlation with the previous state. Formula 3 shows the relationship between the moving velocities and the directions in BAM.

$$
\begin{aligned}
& v(t+\Delta t)=\min \left[\max (v(t)+\Delta t, 0), v_{\max }\right] \\
& \theta(t+\Delta t)=\theta(t)+\Delta \theta
\end{aligned}
$$

Where $V_{\max }$ is the maximum velocity of the MN; $\Delta t$ is the time increment; $v(t)$ and $\boldsymbol{\theta}(t)$ are the velocity and the moving direction at the moment of $t$, respectively. The probability distribution of nodes' mobile direction at the next moment is shown in Fig. 2. Where, $\mathbf{p}_{\mathbf{i}}$ indicates the probability of the direction consisting with the velocity at this moment and $\mathrm{p}_{0}$ denotes the largest probability among them, which means that the current 
nodes' direction remains unchanged. Then, formulation 4 can be deduced.

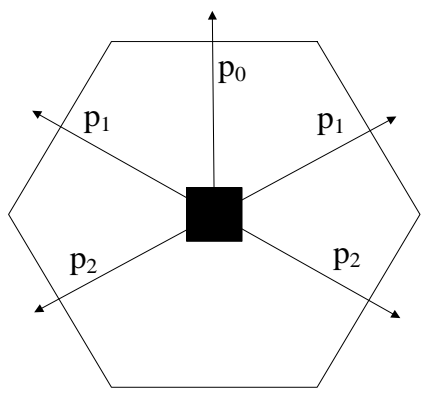

Fig. 2. Probability distribution of direction.

$$
p_{0}+2 p_{1}+2 p_{2}=1
$$

\subsection{Nodes Working State}

A MN's state list $F_{i}$ is active or idle and can be formulated in 2-4. Suppose that the service arrival meets the Poisson distribution with parameter $\lambda$ at any moment and the service cost meets the exponential distribution with parameter $\mu$. Thus, the average service time and the average arrival time are $1 / \mu$ and $1 / \lambda$, respectively.

$$
F_{i}=\left\{\begin{array}{ll}
1, & \text { active } \\
0, & \text { idle }
\end{array}\left(i=1, \ldots, N_{\text {total }}\right)\right.
$$

Here $N_{\text {total }}$ is the total node number in the system; $N_{a c t}$ and $N_{\text {idl }}$ are the numbers of active and idle nodes, respectively. The service time $t_{\text {act }}$ and the interval time $t_{\text {idl }}$ are random variables satisfying the exponent distribution with the average values of $1 / \mu$ and $1 / \lambda$, respectively [18]. The relay handover probability density functions (PDFs) $f_{T}\left(t_{\text {act }}\right)$ and $f_{T}\left(t_{\text {idl }}\right)$ are expressed as:

$$
\begin{aligned}
& f_{T}\left(t_{\text {act }}\right)=\left\{\begin{array}{cc}
\mu e^{-\mu t_{a c t}}, & t_{\text {act }}>0 \\
0 & , \text { other }
\end{array}\right. \\
& f_{T}\left(t_{\text {idl }}\right)=\left\{\begin{array}{cl}
\mu e^{-\mu t_{i d l}}, t_{\text {idl }}>0 \\
0, & \text { other }
\end{array}\right.
\end{aligned}
$$

\section{Mobile Relay Selection Based on Bi-Target Selection}

In MCNs, the system throughput and the switching frequency are essential, and a maximal throughput and minimal switching are expected. Both indicators are affected by the communication state, the velocity and the relative position of the alternative RN [19]. 


\subsection{Switching Prediction in City Micro-Cell}

While analyzing the relative motion between the MNs and the RNs, the switching probability can be estimated combined with the probability in shadow boundary. The BS, the nodes and the relays have the same parameters as mentioned above and are shown in Fig. 3. In initial time, the velocities of the $M N_{1}$ and the selected $R N_{1}$ in the shadow area are $\left(v_{m}, \theta_{m}\right)$ and $\left(v_{r}, \theta_{r}\right)$, respectively; $D_{\text {OM}}(t)$ is the distance from the double-hop user to the BS at time $t$ and at the same time, $D_{O R}(t), D_{S M}(t), D_{S R}(t)$ and $D_{R M}(t)$ are the distances from the $R N_{1}$ to the BS, from the $M N_{1}$ to the shadow center, from the $R N_{1}$ to the shadow center and from the $R N_{1}$ to the $M N_{1}$, respectively. $D_{S R}(t), D_{S M}(t)$ and $D_{R M}(t)$ forms a triangle at time t. $V_{m}, V_{r}$ are the velocities of the $M N_{1}$ and the $R N_{1}$, respectively.

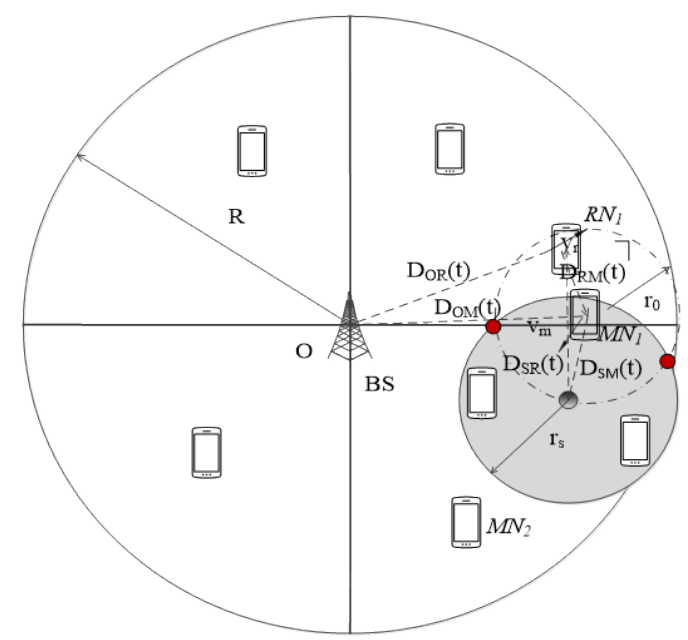

Fig. 3. Dynamic scene in city microcell.

After $\Delta \boldsymbol{t}$, the relative motion including the velocity and the distance between the $M N_{1}$ and the $R N_{1}$ is shown in formula 8, 9:

$$
\begin{aligned}
& \begin{cases}x_{m}(t+\Delta \mathrm{t})=v_{\mathrm{m}}(t) \Delta t \cos \theta_{m}(t), & y_{m}(t+\Delta \mathrm{t})=v_{m}(t) \Delta t \sin \theta_{m}(t) \\
x_{r}(t+\Delta \mathrm{t})=v_{\mathrm{r}}(t) \Delta t \cos \theta_{r}(t), & y_{r}(t+\Delta \mathrm{t})=v_{\mathrm{r}}(t) \Delta t \sin \theta_{r}(t)\end{cases} \\
& D_{O M}(\mathrm{t}+\Delta \mathrm{t})=\sqrt{x_{m}(t+\Delta \mathrm{t})^{2}+y_{m}(t+\Delta \mathrm{t})^{2}} \\
& D_{O R}(\mathrm{t}+\Delta \mathrm{t})=\sqrt{x_{r}(t+\Delta \mathrm{t})^{2}+y_{r}(t+\Delta \mathrm{t})^{2}} \\
& D_{R M}(\mathrm{t}+\Delta \mathrm{t})=\sqrt{\left(x_{r}(t+\Delta \mathrm{t})-x_{m}(t+\Delta \mathrm{t})\right)^{2}+\left(y_{r}(t+\Delta \mathrm{t})-y_{m}(t+\Delta \mathrm{t})\right)^{2}} \\
& D_{S M}(\mathrm{t}+\Delta \mathrm{t})=\sqrt{\left(x_{m}(t+\Delta \mathrm{t})-x_{s}(t)\right)^{2}+\left(y_{m}(t+\Delta \mathrm{t})-y_{s}(t)\right)^{2}} \\
& D_{S R}(\mathrm{t}+\Delta \mathrm{t})=\sqrt{\left(x_{r}(t+\Delta \mathrm{t})-x_{s}(t)\right)^{2}+\left(y_{r}(t+\Delta \mathrm{t})-y_{s}(t)\right)^{2}}
\end{aligned}
$$

The $R N_{1}$ is in white region outside the shadow as shown in Fig. 3 . Once the $R N_{1}$ moves 
out of the communication range, the $M N_{1}$ needs to handover the current relay. Meanwhile, the relative position and the communication status between $M N_{1}$ and $R N_{1}$ changes and the following relay switching may occur:

(1) In the first case, the $M N_{1}$ has changed its location and it has moved out of the BS scope into the neighbor cells or out of the shadow area into the BS central area. Therefore, the $M N_{1}$ can communicate with the BS directly without a relay. Thus, formula (10) can be obtained.

$$
D_{O M}(t)<R
$$

(2) The second case is that the current relay $R N_{1}$ has moved out of the node's area and into the shadow region. Therefore, the $M N_{1}$ reselects another relay.

$$
\left\{\begin{array}{l}
D_{S R}(t)<r_{s} \\
D_{R M}(t)>r_{0}
\end{array}\right.
$$

(3) The third case is that the $M N_{1}$ is hanged up with its communication from active to idle and $R N_{1}$ is released.

(4) The last case is that the $R N_{1}$ becomes busy. When the service is coming, the $R N_{1}$ is changed from idle to active and the candidate relay is re-selected.

The relay handover is triggered in the second and the fourth case. In both cases, the relay is reselected. After a short time interval $t$, the coordinates of the $M N_{1}$ and the $R N_{1}$ are $\left(x_{m}+v_{m} t \cos \theta_{m}, \quad y_{m}+v_{m} t \sin \theta_{m}\right) \quad$ and $\left(x_{r}+v_{r} t \cos \theta_{r}, y_{r}+v_{r} t \sin \theta_{r}\right) \quad$ respectively. Suppose that the $R N_{1}$ comes into the shadow area at time $t_{1}$ and locates in the $M N_{1}$ communication scope at time $t_{2}$. Then, the distance from the $R N_{1}$ to the shadow center is $D_{S R}\left(t_{1}\right)=r_{s}$ at time $t_{1}$ according to the formula 11 .

Combining the results of 8 and $9, D_{S R}\left(t_{1}\right)=r_{s}$ can be replaced by the following:

$$
\sqrt{\left(x_{\mathrm{r}}-x_{s}+v_{r} t_{1} \cos \theta_{r}\right)^{2}+\left(y_{r}-y_{s}+v_{r} t_{1} \sin \theta_{r}\right)^{2}}=r_{s}
$$

Solving the equation as:

$$
v_{r}^{2} t_{1}^{2}+2 v_{r}\left(\left(x_{r}-x_{s}\right) \cos \theta_{r}+\left(y_{r}-y_{s}\right) \sin \theta_{r}\right) t_{1}+\left(\left(x_{r}-x_{s}\right)^{2}+\left(y_{r}-y_{s}\right)^{2}-r_{s}^{2}\right)=0
$$

From the above quadratic equations, $t_{1}$ has a positive and a negative solution:

$$
t_{1}=-\left(\left(x_{\mathrm{r}}-x_{s}\right) \cos \theta_{r}+\left(y_{r}-y_{s}\right) \sin \theta_{r}\right) / v_{r} \pm \sqrt{r_{s}^{2}-\left(\left(x_{\mathrm{r}}-x_{s}\right) \sin \theta_{r}-\left(y_{r}-y_{s}\right) \cos \theta_{r}\right)^{2}} / v_{r}
$$


The intersecting region of relay's scope and cell must have two cross points on the edge of the shaded region (see red points in Fig. 4). The nearest cross point is the critical value into the shadow area. Therefore, $t_{1}$ should take the smaller root from the two equation roots (or solutions).

Similarly, $t_{2}$ can be obtained from $D_{R M}\left(t_{2}\right)=r_{0}$ as:

$$
\sqrt{\left(x_{\mathrm{r}}-x_{m}+v_{\text {relative }} t_{2} \cos \theta_{\text {relative }}\right)^{2}+\left(y_{r}-y_{m}+v_{\text {relative }} t_{2} \sin \theta_{\text {relative }}\right)^{2}}=r_{0}
$$

The $R N_{1}$ 's relative speed to $M N_{1}$ is $\left(v_{\text {rel }}, \theta_{\text {rel }}\right)$, its relative coordinate to $M N_{1}$ is

$$
\left(x_{\text {rel }}, y_{\text {rel }}\right) \text {. }
$$

$$
\begin{gathered}
x_{r e l}=x_{r}-x_{m} \\
y_{r e l}=y_{r}-y_{m} \\
v_{r e l}=\sqrt{\left(v_{\mathrm{r}} \cos \theta_{\mathrm{r}}-v_{\mathrm{m}} \cos \theta_{\mathrm{m}}\right)^{2}+\left(v_{\mathrm{r}} \sin \theta_{\mathrm{r}}-v_{\mathrm{m}} \sin \theta_{\mathrm{m}}\right)^{2}} \\
\theta_{r e l}=\arctan \left(\frac{v_{\mathrm{r}} \sin \theta_{\mathrm{r}}-v_{\mathrm{m}} \sin \theta_{\mathrm{m}}}{v_{\mathrm{r}} \cos \theta_{\mathrm{r}}-v_{\mathrm{m}} \cos \theta_{\mathrm{m}}} \pm \pi\right.
\end{gathered}
$$

Using the above-mentioned method to solve the equation, $t_{2}$ should take the positive root since the relay's left time within the intersecting region is higher. $t_{2}$ is as follows:

$$
\begin{aligned}
& t_{2}=-\left(\left(x_{r}-x_{m}\right) \cos \theta_{r e l}+\left(y_{r}-y_{m}\right) \sin \theta_{r e l}\right) / v_{r e l} \\
& +\sqrt{r_{0}^{2}-\left(\left(x_{r}-x_{m}\right) \sin \theta_{r e l}-\left(y_{r}-y_{m}\right) \cos \theta_{r e l}\right)^{2}} / v_{r e l}
\end{aligned}
$$

The maintain time that $R N_{1}$ stays in the intersecting region of its scope and unshaded region is $t_{m a \mathrm{i}}=\min \left(t_{1}, t_{2}\right)$. The handover occurs when the $R N_{1}$ moves out of the intersecting region. So, the $R N_{1}$ 's activation time is greater than maintain time. According to 2-3, 2-5 and 2-6, the probability of $P_{\text {han }}{ }^{(1)}$ is express as follows:

$$
\begin{aligned}
P_{\text {han }}^{(1)}=P\left(t_{\text {act }}>t_{\text {mai }}\right) & \\
= & \int_{\text {mi }}^{\infty} \mu e^{-\mu_{\text {act }}} d t_{\text {active }} \\
= & e^{-\mu t_{\text {mai }}}
\end{aligned}
$$

During the communication process of $M N_{1}$, the service/call arrives and relay handover 
occurs. So, the MN's communication time is longer than idle time. The probability of $P_{\text {han }}{ }^{(1)}$ is express as follows:

$$
\begin{aligned}
P_{\text {han }}{ }^{(2)}= & P\left(t_{\text {act }}>t_{\text {idl }}\right) \\
& =\int_{0}^{\infty} \int_{t_{\text {didl }}}^{\infty} \mu e^{-\mu t_{\text {cati }}} \lambda e^{-\lambda t_{\text {till }}} d t_{\text {act }} d t_{\text {idl }} \\
& =\frac{\lambda}{\lambda+\mu}
\end{aligned}
$$

According to the distribution of service/call and switching probabilities in above formula, the whole handover probability can be described as:

$$
\begin{aligned}
P_{\text {han }}=P_{\text {han }}{ }^{(1)}+P_{\text {han }}{ }^{(2)} & \\
= & P\left(t_{a c t}>t_{\text {mai }}\right)+P\left(t_{a c t}>t_{i d l}\right) \\
& =\frac{\lambda}{\lambda+\mu}+e^{-\mu t_{\text {mai }}}
\end{aligned}
$$

Using 23, the derivative of time is obtained as:

$$
\begin{aligned}
P_{\text {han }}\left(t_{\text {mai }}\right)=\frac{d P_{\text {han }}}{d t_{\text {mai }}} & \\
= & -\mu e^{-\mu t_{\text {mai }}}
\end{aligned}
$$

According to for 24 , reducing the switching should reduce $t_{\text {mai }}$. Using the method proposed in [2] to calculate the predicted value of $t_{m a \mathrm{i}}$, then, the switching prediction $k_{\text {han }}(t)$ of candidate $R N_{k}$ at time $t$ is as follows:

$$
k_{\text {han }}(t)=\frac{T_{\text {mai }} k(t)}{\frac{1}{n} \sum_{i=1}^{n} T_{\text {mai }} i(t)}
$$

where $T_{m a \mathrm{i}} k(t)$ is the maintain time of the candidate $R N_{t}$ at time $t$, and $\sum_{i=1}^{n} T_{m a \mathrm{i}} i(t)$ is the whole maintain time of candidate relays at time $t$.

\subsection{System Throughput Prediction}

The system throughput is proportional to the current relay link transmission rate, but is inversely associated with other candidate relay link transmission rates. Therefore, the higher the transmission rate is, the more relay selection probability will be.

The link transmission rate can be calculated through the Shannon expression [20] [21]: 


$$
\begin{aligned}
& \gamma_{A L}=B_{A L} \cdot \log _{2}\left(1+S I N R_{A L}\right) \\
& \gamma_{B L}=B_{B L} \cdot \log _{2}\left(1+S I N R_{B L}\right)
\end{aligned}
$$

let $\gamma_{A L}=\gamma_{B L}=\gamma_{i}, \gamma_{i}$ is RN's transmission rate, then the balance throughput should be obtained and the transmission rate of the selecting relay $\mathrm{k}$ and the average transmission rate in the RN set are $\gamma_{k}, \gamma_{\text {ave }}=\frac{1}{n} \sum_{i=1}^{n} \gamma_{i}$, respectively. The system throughput prediction takes the average transmission rate instead of selecting best one as the CSI factor in the RN set [27]. So, at moment of $t$, the throughput prediction of selecting relay $k$ can be expressed as:

$$
k_{t h r}(t)=\frac{\gamma_{k(t)}}{\frac{1}{n} \sum_{i=1}^{n} \gamma_{i(t)}}
$$

\section{The MN Selection Model Based on Bi-Target Selection}

Both the throughput and the switching affect the relay selection and the switching probability is correlated with the RN's activation time in the intersecting region. The longer activation time is, the more relay selection probability will be. These logarithms are different for throughput prediction and switching prediction (see formula 3-16, 3-17 and 3-19, 3-20). For normalization, the judge-function for relay selection can be constructed:

$$
F_{k(t)}=\alpha \log k_{t h r}(t)+\beta \log k_{h a n}(t)
$$

In the above formula, $\alpha$ and $\beta$ are the adjustment coefficients with $\alpha+\beta=1$. The relay with maximal $F_{k(t)}$ will be selected as the current relay. In essence, $F_{k(t)}$ is a dimensionless value. The selection strategy of the algorithm can be adjusted by changing $\alpha$ and $\beta$. For example, when $\alpha=0$, the algorithm degrades into selection algorithm based on the switching probability with $k_{t h r}(t)$ as the throughput function that is equal to $\frac{\gamma_{k(t)}}{\frac{1}{n} \sum_{i=1}^{n} \gamma_{i(t)}}$ using 3-20. Whereas, when $\beta=0, \quad k_{\text {han }}(t)$ is $\frac{T_{m a \mathrm{i}} k(t)}{\frac{1}{n} \sum_{i=1}^{n} T_{m a \mathrm{i}} i(t)}$ using 3-18.

The algorithm implementation process is as follows: 
(1) Set the SINR threshold $\gamma_{t h}$.

(2) At the initial state, the practical SINR should be calculated from the path loss in accordance to the user request.

(3) According to the SINR and the threshold $\gamma_{\text {th }}$, the MNs can be divided into one-hop and two-hop nodes/users.

(4) The one-hop node/user communicates directly with the base station, while the two-hop node/user sends signal to the users around to select the alternative relay based on $\gamma_{\text {th }}$.

(5) The alternative relay sends the ACK signal, the two-hop receives it and adds the relay information into the relay set $R\left(Q_{i}, \quad i=1, \ldots, M\right)$.

(6) The users' radius $r_{0}$ and the other information are calculated according to formula 4-1 and then the best relay is selected.

After that, the two-hop user can select the best relay based on the bi-target including the switching probability and the system throughput. The proposed algorithm improves the system throughput and reduces the switching probability while simultaneously balancing the bandwidth of AL and BL in the dynamic scenario.

\section{Simulation Results}

In this paper, MATLAB is used to simulate the MCN's dynamic environment. The main parameters are: shadow radius $=150 \mathrm{~m}$, link radius $=[100,300]$, average service time $1 / \mu$ $=100 \mathrm{~s}$, idle time $=1000 \mathrm{~s}$ and the number of users in micro cell $=[50,500]$. In the simulation process, the $\mathrm{MN}$ number, the maximal velocity and the communication radius are varied and the proposed algorithm is compared with bi-target selection and other algorithms in terms of the system throughput and the handover. The other algorithms include the shortest path algorithm [22], the traditional BRS algorithm [23,23] and the handover/switching probability algorithm [25]. In the shortest path scheme, the AL and the BL are labeled as $D_{M N}$ and $D_{O N}$, and the relay with the $\min \left\{D_{O N}+D_{\mathrm{MN}}\right\}$ is selected. The traditional BRS (Bidirectional Relay Selection) algorithm selects the relay according to the two-way SINR and channel Gain. The handover algorithm is introduced in the proposed algorithm when $\alpha=0$. 


\subsection{Switching with Single Factor}

The Switching/handover is observed by only taking the MN number into account in the simulation. When the maximal velocity is $15 \mathrm{~m} / \mathrm{s}$ and the communication coverage is $150 \mathrm{~m}$, the number of handover in the micro cell shadow is shown in Fig. 4:

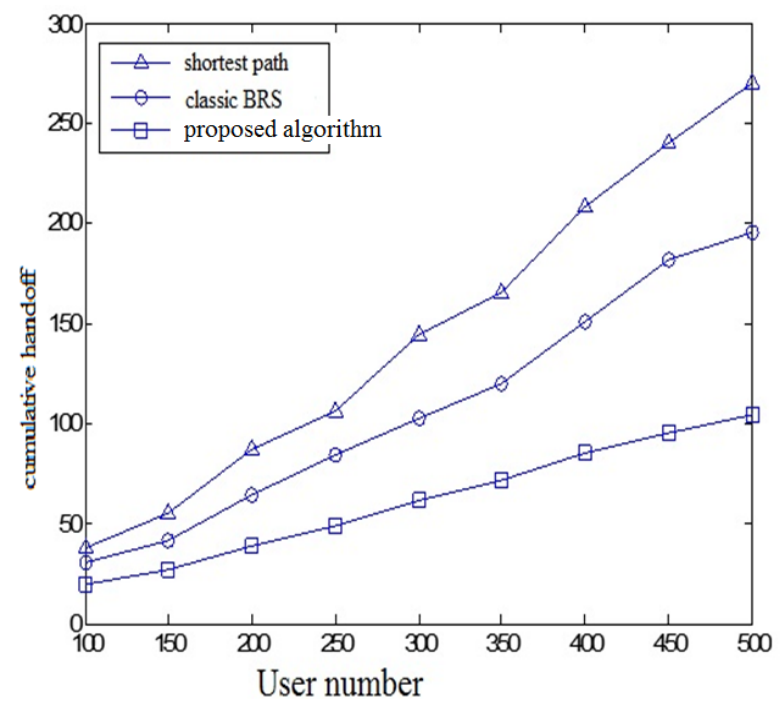

Fig. 4. Cumulative handover under 3 algorithms.

Usually, the handover increases with the MNs number. However, the proposed algorithm has successfully reduced the handover number by using the handover probability prediction in the dynamic scene. The handover probability algorithm should be excluded in the experiment.

\subsection{System Throughput under Multiple Parameters}

The simulations in this section consider the system throughput and the handover by comparing the four different algorithms mentioned above: the shortest path algorithm, the traditional BRS algorithm, the handover probability algorithm and the proposed algorithm. 


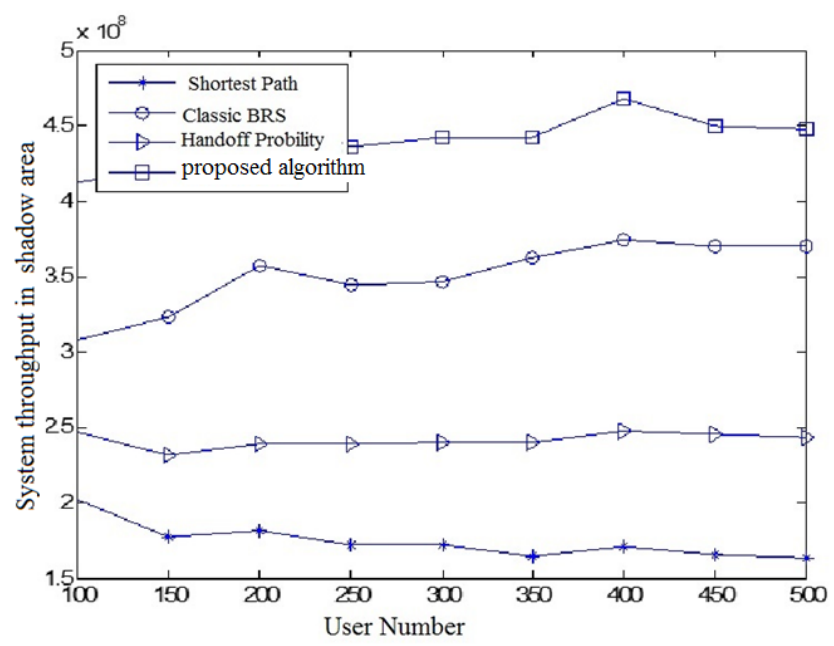

Fig. 5. Throughput under 4 algorithms.

The throughput changes with the MN/user number, the maximal velocity and the communication coverage. When the maximal velocity is $15 \mathrm{~m} / \mathrm{s}$, the communication coverage is $150 \mathrm{~m}$ and $\alpha=0.7$, the user throughput changes with the MN/user number. But, the system has a fixed bandwidth and bandwidth of each MN decreases with the increase of MN number. Therefore, the system throughput remains unchanged. The proposed algorithm has higher throughput and quantity of experience (QoE) than the other three algorithms as shown in Fig. 6.

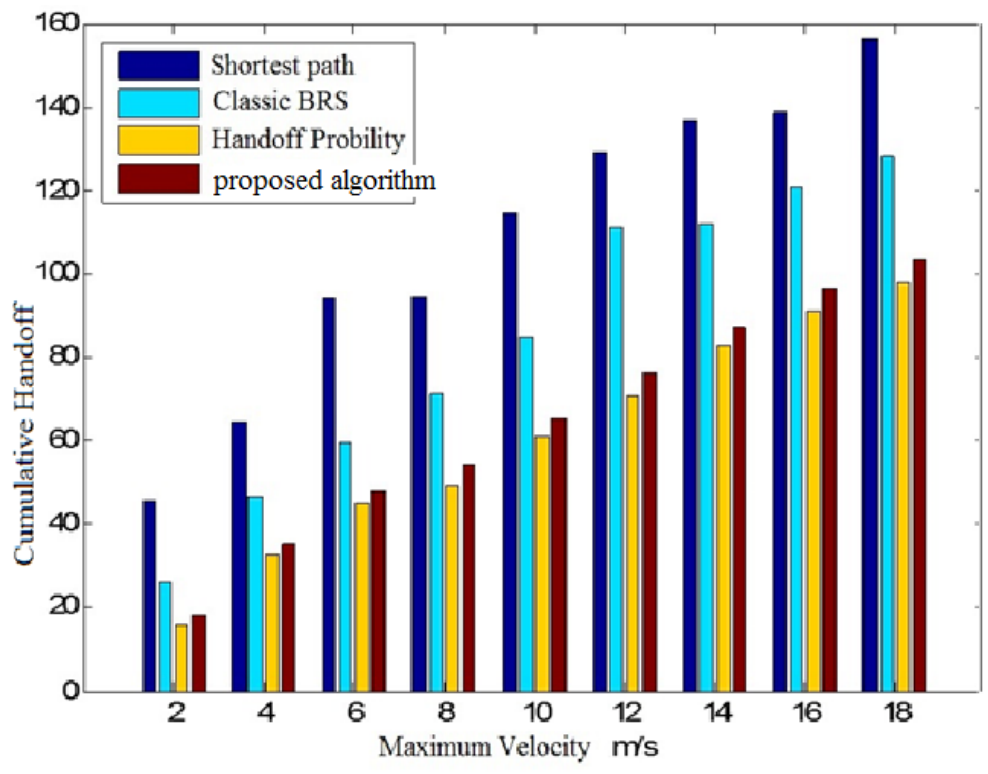

Fig. 6. Handover number in 4 algorithms. 
When the parameters remain unchanged, the number of handover changes with the MN number (see Fig 6). The general trend of handover is increasing with the MN number. Both the shortest path and the BRS algorithms have large handovers because they do not predict the MN location and its mobile status. Whereas, the other two algorithms have better performance. Moreover, the handover probability algorithm has the smallest handover because it is similar to the proposed algorithm with $\alpha=0$.

\subsection{Under the Condition of MN Velocity}

In order to analyze the system throughput under the condition of changing $\mathrm{MN}$ velocity, the performances of the four algorithms with different velocities are compared in Fig 7. The system throughput does not change significantly with MNs velocity especially in the shortest path algorithm and the handover probability algorithm. The proposed algorithm has higher throughput in the beginning, which then decreases with the increase of MN velocity because the MNs maintain time affects the handover prediction according to the formula 3-18.

Moreover, the handover number is increasing with the increase of the MN velocity in Fig. 8. Since, the communication coverage of the relay is fixed, the faster the MN moves, the more the handover probability will be. Thus, the shortest path and the BRS algorithms have larger handover numbers. The handover probability algorithm has the smallest handover number, which is the optimization goal of the algorithm proposed in this paper.

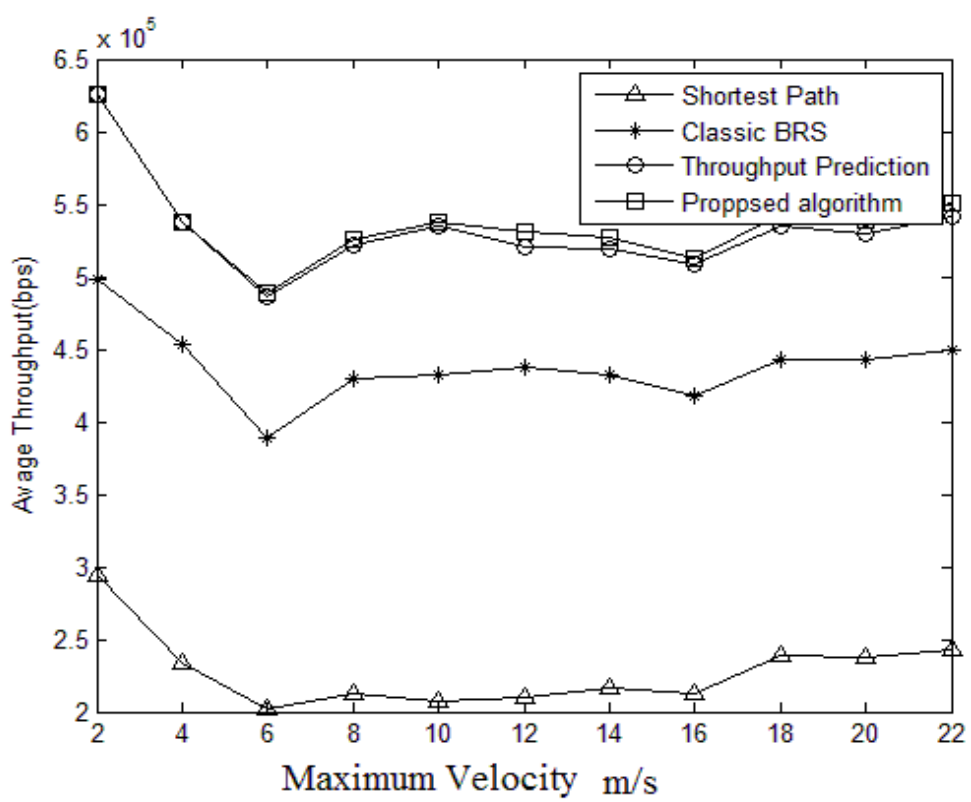

Fig. 7. System throughput under 4 algorithms. 


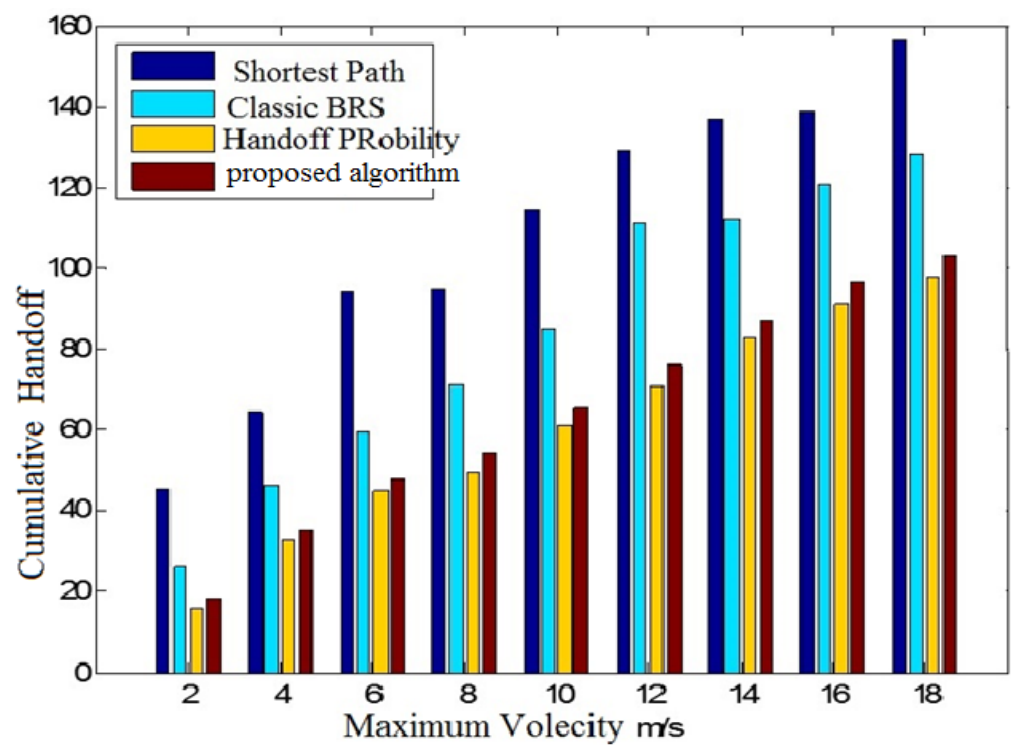

Fig. 8. Handover number under 4 algorithms.

\subsection{Under the Condition of Communication Coverage}

In this section, the system throughput under the condition of changing MNs communication distance or coverage using 4 different algorithms is analyzed. The MN number is 200, $V_{\max }=10 \mathrm{~m} / \mathrm{s}$ and $\alpha=0.7$.

According to Fig. 9, the total throughput in shadow area is decreasing with the increase of MN communication coverage because the access link is the bottleneck. With the increase of communication coverage, some two-hop MNs will not require the handover anymore, so the communication quality is slightly decreased. The mobile relay selection algorithm based on the bi-target selection has the largest throughput, the BRS algorithm takes the second place and the shortest path algorithm has the lowest throughput. The performance of the handover probability algorithm is between the BSR and the shortest path algorithms. 


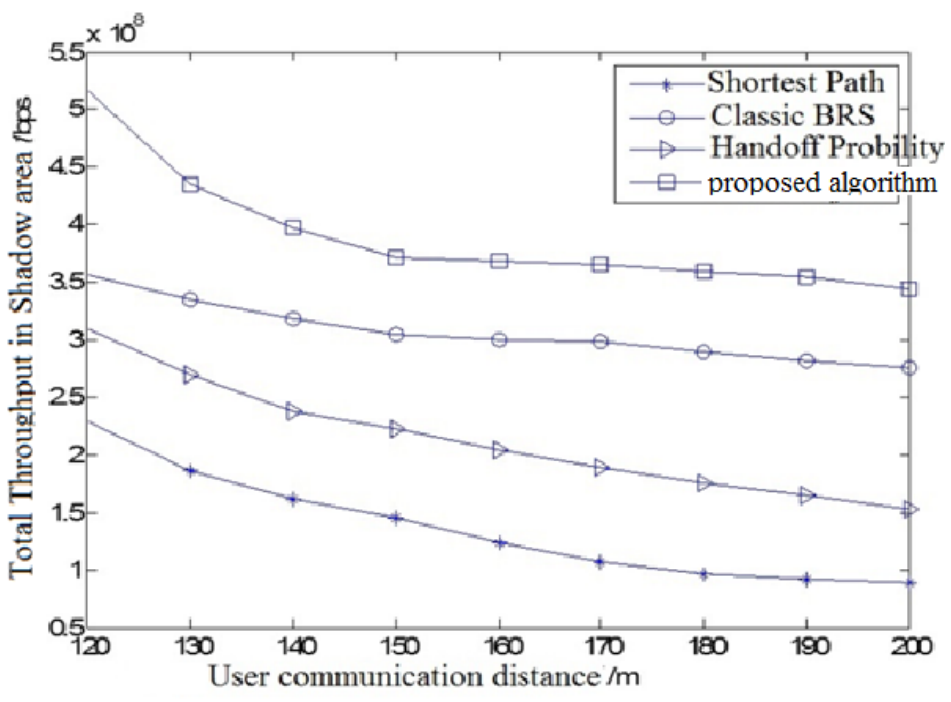

Fig. 9. Throughput under 4 algorithms.

\section{Conclusion and further research}

In this paper, a mobile relay selection scheme based on the bi-target selection under the dynamic scene is proposed to effectively improve the system throughput and reduce the switching. The expressions are derived for the bi-target and relay motion patterns are used in the dynamic scene to select the appropriate RN. The proposed scheme analyzes the work status of the MNs and the RNs, and predicts the system throughput and handover probabilities. Then the relay selection function is implemented based on the bi-target selection mechanism. The validity of the proposed scheme has been verified by comparing its performance with three traditional algorithms under the dynamic simulation environment build by using MATLAB toolbox.

\section{References}

[1] Wang L, Peng T and Yang Y, et al., "Interference Constrained Relay Selection of D2D Communication for Relay Purpose Underlaying Cellular Networks," in Proc. of IEEE Wireless Communications, Networking and Mobile Computing (WiCOM), pp.1-5, September, 2012.

Article (CrossRef Link).

[2] Zhang H, Hong P, Xue K., "Mobile-based relay selection schemes for multi-hop cellular networks," Communications and Networks, vol. 15, no.1, pp.45-53, Journal, 2013.

Article (CrossRef Link)

[3] Chunguo Li, Luxi Yang and Wei-Ping Zhu, "A two-way MIMO relaying scheme with partial channel state information,” Wireless Personal Communications, vol. 72, no. 4, pp.1949-1968, April. 2013. Article (CrossRef Link)

[4] Laneman J N, Tse D N C, Wornell G W., "Cooperative diversity in wireless networks: Efficient protocols and outage behavior," IEEE Transactions on Information theory, vol. 50, no.12, pp 3062-3080, December, 2004. Article (CrossRef Link) 
[5] Chunguo Li, Luxi Yang, and Wei-Ping Zhu, "Minimum mean squared error design of single-antenna two-way distributed relays based on full or partial channel state information," IET Communications, vol.5, no.5, pp.728-735, March, 2011. Article (CrossRef Link)

[6] Zou Y, Zhu J, Zheng B, et al., "An adaptive cooperation diversity scheme with best-relay selection in cognitive radio networks," IEEE Transactions on Signal Processing, Vol.58, no.10, pp.5438-5445, October, 2010. Article (CrossRef Link)

[7] Yu C H, Doppler K, Ribeiro C B, et al., "Resource Sharing Optimization for Device-to-Device Communication Underlying Cellular Networks,” IEEE Transactions on Wireless Communications, Vol.10, no.8, pp.2752-2763, Augest 2011. Article (CrossRef Link)

[8] D. S. Michalopoulos, H. A. Suraweera, G. K. Karagiannidis, and R. Schober, “Amplify and forward relay selection with outdated channel estimates,” IEEE Trans. Commun., vol. 60, no. 5, pp. 1278-1290, May 2012. Article (CrossRef Link)

[9] Xing X, Jing T, Cheng W, et al., "Spectrum prediction in cognitive radio networks," IEEE Wireless Communications, vol.20 ,no.2, pp.90-96, February, 2013. Article (CrossRef Link)

[10] Ding G, Wang J, Wu Q, et al., "On the limits of predictability in real-world radio spectrum state dynamics: from entropy theory to 5G spectrum sharing,” IEEE Communications Magazine, vol.53, no.7, pp.178-183, July, 2015. Article (CrossRef Link)

[11] Ding G, Wang J, Wu Q, et al., "Cellular-Base-Station-Assisted Device-to-Device Communications in TV White Space,” IEEE Journal on Selected Areas in Communications, vol.34, no. 1, pp.107-121, January,2015. Article (CrossRef Link)

[12] Tehrani M N., Uysal M.,and Yanikomeroglu H., "Device-to-Device Communication in 5G Cellular Networks: Challenges, Solutions, and Future Directions,” IEEE Communications Magazine, vol.52,no.5, pp. 86-92, 2014. Article (CrossRef Link)

[13] ZHU Jia , ZHENG Bao-yu , ZOU Yu-long. "Cooperative Spectrum Sensing in Multiuser Cognitive Radio Networks with Best Relay Selection,” Acta Electronica Sinca, vol. 38, no.1, pp.92-98, 2010. Article (CrossRef Link)

[14] L. C. Wang, W. S. Su, J. H. Huang, A. Chen, and C. J. Chang, "Optimal relay location in multi-hop cellular systems,” in Proc. of IEEE Wireless Communications and NETWORKING Conference, pp.1306-1310, 2008. Article (CrossRef Link)

[15] Chunguo Li, Peng Liu, Chao Zou, Fan Sun, John M. Cioffi, and Luxi Yang, "Spectral-efficient cellular communications with coexistent one- and two-hop transmissions," IEEE Transactions on Vehicular Technology, vol. 65, no. 8, pp. 6765-6772, Aug. 2016. Article (CrossRef Link)

[16] 3GPP. Evolved Universal Terrestrial Radio Access (E-UTRA); Radio Frequency (RF) system scenarios. Release 9(S), 3GPP, TR 36.942 V9.0.1, 2010.

[17] Neal P, Roberts G. "Optimal Scaling Of Random Walk Metropolis Algorithms With Non-Gaussian Proposals,” Methodology \& Computing in Applied Probability, vol. 13, no.3, pp. 583-601, 2011. Article (CrossRef Link)

[18] Cui H, Song L, Jiao B. "Weighted Bidirectional Relay Selection for Outdated Channel State Information,” IEEE Transactions on Communications, vol.62, no.2, pp.500-509, 2014. Article (CrossRef Link)

[19] Zhu K, Niyato D, Wang P., "Dynamic Service Selection and Bandwidth Allocation in IEEE 802.16m Mobile Relay Networks,” IEEE Journal on Selected Areas in Communications, 2012, vol.30, no.9, pp.1798-1805, 2012. Article (CrossRef Link)

[20] Jung Man Hong, Jong Hyup Lee and Soong-Hee Lee, "Optimal Base Station Clustering for a Mobile Communication Network Design," KSII Transactions on Internet and Information Systems, vol. 5, no. 5, pp. 1069-1084, 2011. DOI: 10.3837/tiis.2011.05.011. Article (CrossRef Link)

[21] L. Song, "Relay selection for two-wa y relaying with amplify-and-forward protocols," IEEE Transactions on Vehicular Technology, vol.60, no.4, pp.1954-1959, 2011. Article (CrossRef Link)

[22] Yuan Zhou, Zesong Fei, Gaishi Huang, Ang Yang and Jingming Kuang, "A Distributed LT Codes-based Data Transmission Technique for Multicast Services in Vehicular Ad-hoc Networks,” KSII Transactions on Internet and Information Systems, vol. 7, no. 4, pp. 748-766, 2013. 
Article (CrossRef Link)

[23] Teyeb O, Phan V V, Raaf B, et al. "Handover framework for relay enhanced LTE networks," in Proc. of IEEE International Conference on Communications Workshop, pp. 1-5, 2009. Article (CrossRef Link)

[24] Ikhlef A, Michalopoulos D S, Schober R., "Max-max relay selection for relays with buffers," IEEE Transactions on Wireless Communications, vol. 11, no.3, pp. 1124-1135, 2012. Article (CrossRef Link)

[25] Pan M S, Lin T M, Chen W T., "An enhanced handover scheme for mobile relays in LTE-A high-speed rail networks," IEEE Transactions on Vehicular Technology, vol.64, no.2, pp. 743-756, 2015. Article (CrossRef Link)

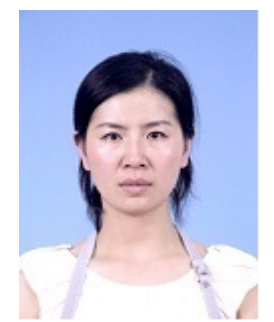

Huijun Dai received the Ph.D. degrees in computer science from the Xi'an Jiaotong University, Xi'an, China, in 2015. She works in the School of Electronic and Information Engineering in Xi'an Jiaotong University as a teacher since 2015. Her research interests include D2D Communication, QoS routing, and Privacy Preserving.

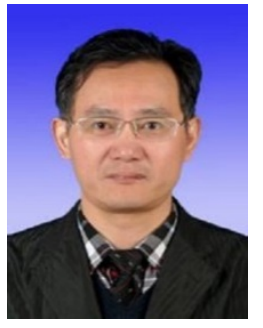

Xiaolin Gui received the B.S. degree in computer and M.S. and Ph.D. degrees in computer science from the Xi'an Jiaotong University of China, Xi'an, China, in 1993 and 2001, respectively. He is a Professor and serves as a Deputy Dean of the School of Electronic and Information Engineering, Xi'an Jiaotong University, Xi'an, China, since 2013. He is also the Director of the Key Laboratory of Computer Network of Shaanxi Province (CNKL) and the Center for Grid and Trusted Computing (CGTC), Xi'an Jiaotong University, since 2008. He has authored many leading papers in international journals and conferences. His research interests include secure computation of open network systems (include grid, P2P, cloud), dynamic trust management theory, and development on community network. Prof. Gui has served as a Program Committee Member for numerous international conferences such as IEEE iThings2013, NCSC2012, ISCTSC2012, HOWAN2009, CollaborateCom2009, and GCC2004. He was the recipient of the New Century Excellent Talents Award from the University of China in 2005. 


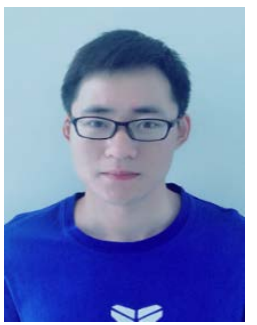

Zhaosheng Dai was born in 1993. He received the B.S. degree in computer science from Chongqing University, Chongqing, China, in 2015. He is currently working toward the M.S. degree in computer science and technology at Xi'an Jiaotong University, Xi’an, Shaanxi, China. His research Interests include Network Security and Privacy Protection.

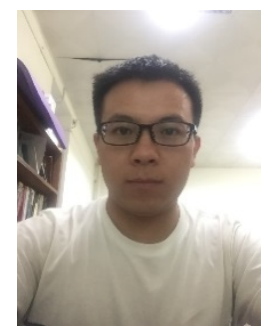

Dewang Ren was born in 1989. He received the B.S. degree from Lanzhou Jiaotong University, Lanzhou, Gansu, China, in 2012 and M.S. degree from Beijing Jiaotong University, Beijing, China, in 2015. He is currently working toward the $\mathrm{Ph} . \mathrm{D}$. degree in computer science and technology at Xi'an Jiaotong University, Xi'an, Shaanxi, China. His rese arch Interests include Mobile Edge Computing and Cloud Computing

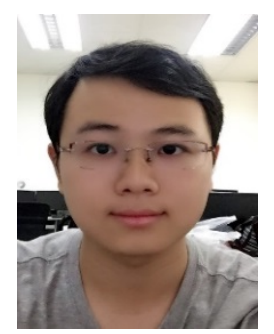

Yingjie Gu was born in 1992. He received the B.S. degree in computer science and technology from Northwestern Polytechnical University, Xi’an, Shaanxi, China, in 2015. He is currently working toward the PH.D. degree in computer science and technology at Xi'an Jiaotong University, Xi'an, Shaanxi, China. His research Interests include Network Security, Blockchain and Cloud Computing. 